- Ultrafine resistance spot welding particles with similar composition and number concentration but lower mass concentration do not induce such reactions.

- Both, copper and zinc are individually able to induce such inflammation reactions. However, copper seems to have a higher potential for this induction.

- The inflammatory reaction is also reflected by an increase of Serum Amyloid A (SAA) and Interleukin 6 in the blood and by an increase of Interferon- $\lambda$ and CRP in nasal secretions.

Discussion Since increases of CRP, SAA, and IL-6 indicate an increased risk for cardiovascular disease, exposure to zinc and copper containing welding fumes may have to be considered for the prevention of work related cardiovascular disease. Future studies should investigate, if the observed inflammatory reaction persists after repeated exposure

\section{THE ASSOCIATION OF BLOOD LEAD LEVEL AND SERUM LIPID CONCENTRATIONS MAY BE MODIFIED BY METALLOTHIONEIN 2A POLYMORPHISMS}

1,2,3,4,5 Chen-Cheng Yang ${ }^{5}$ Chih-Shien Chuang, 1,2,3,4 Chia-1 lin ${ }^{3}$ Chao-ling Wang, ${ }^{6} Y$ ungCheng Huang, ${ }^{3,5,7}$ Hung-Yi Chuang. 'Health Management Centre, Kaohsiung Medical University Hospital, Kaohsiung, Taiwan; ${ }^{2}$ Health Management Centre, Kaohsiung Municipal Ta-Tung Hospital, Kaohsiung, Taiwan; ${ }^{3}$ Department of Environmental and Occupational Medicine, Kaohsiung Medical University Hospital, Kaohsiung, Taiwan; ${ }^{4}$ Department of Environmental and Occupational Medicine, Kaohsiung Municipal Ta-Tung Hospital, Kaohsiung, Taiwan; ${ }^{5}$ Graduate Institute of Medicine, College of Medicine, Kaohsiung Medical University, Kaohsiung, Taiwan; ${ }^{6}$ Department of Family Medicine, Pingtung Christian Hospital, Pingtung, Taiwan; 'Department of Public Health, College of Health Sciences, Kaohsiung Medical University, Kaohsiung, Taiwan

\subsection{6/oemed-2018-ICOHabstracts. 1448}

Introduction Lead in blood can stimulate lipid oxidation in phosphatidylcholine and increase peroxidation in lipids. Metallothionein (MT) is a cysteine rich protein that can influence the detoxification of heavy metals and scavenge oxidative stress for free radicals. One of the most expressive functional genes in humans is the MT2A gene.

This study aims to determine if the association of the blood lead level and lipid biomarkers was influenced by MT2A polymorphisms.

Methods We recruited 677 participants after informed consent was obtained. All of the samples collected were analysed for lipid biomarkers and blood lead levels and were genotyped for MT2A polymorphisms by RT-PCR. A short questionnaire collected the medical history and alcohol and cigarette consumption information. The data were used for descriptive analyses and linear regression models.

Result The investigation revealed that lead elevated concentration increased low-density lipoprotein cholesterol (LDL-C) and decreased high-density lipoprotein cholesterol (HDL-C) by multiple linear models. The carriers of the rs10636 GCrs28366003 AA genetic combination may be less susceptive to lead elevated concentration on HDL-C than other types.

Conclusion In conclusion, the association of the blood lead level and HDL-C may be modified by the MT2A genetic combination: the rs10636 GC-rs28366003 AA genotype could play a protective role in lead elevated concentration on HDL$\mathrm{C}$ in humans.

\section{ACUTE INTOXICATION WITH ARSINE GAS, A CASE REPORT}

${ }^{1} \mathrm{MP}$ Hurtado Pérez*, ${ }^{2} \mathrm{MC}$ Rodríguez Vega, ${ }^{2} \mathrm{ME}$ Aguilar Aldrete, ${ }^{1} \mathrm{GA}$ Real Ornelas. ${ }^{1}$ UIDAC, Research Unit, Teaching and Clinical Support, IMSS, Guadalajara, Mexico; ${ }^{2}$ CUCS, University Centre of health sciences, Guadalajara University, Guadalajara, Mexico

\subsection{6/oemed-2018-ICOHabstracts. 1449}

Introduction Arsine gas is an arsenic compound conjugated with the hydrogen ion generated from several chemical reactions. The arsine gas poisoning is infrequent and usually generated on unexpected reactions from industrial process. Once it`s absorbed into de body, inhibits many enzymatic systems lowing the intracellular glutathione concentration, this causes a oxidative stress damage and hematic, renal, mucosa and erythrocytes cellulary death. Clinically manifests itself with the triad of abdominal pain, oliguria and jaundice, secondary to massive intravascular haemolysis, renal acute injury, hepatic damage and central nervous system injury.

Methods We presented a clinical curse of a patient part of a outbreak of workers exposed to an incidental formation of arsine gas while performance a cadmium extraction process after adding metallic zinc. After a few hours the patient suffered headache, epistaxis, fatigue, mucus dark diarrhoea, vomiting and red urine.

Results The patient evolved to hematuria with posterior oliguria, Generalised muscle weakness, dyspnoea and anasarca. Laboratory test showed urine blood test positive, with blood haemoglobin of $6.6 \mathrm{~g} / \mathrm{dL}$, and an haematocrit of $19.3 \%$, Lactic dehydrogenase of $1469 \mathrm{U} / \mathrm{L}$, blood creatinine: $14.7 \mathrm{mg} / \mathrm{dL}$, total urinary arsenic on 10 th day: $8.6 \mu \mathrm{g} / \mathrm{L}$, with the diagnosis of: massive intravascular haemolysis with acute pre renal injury secondary to arsine gas poisoning. The treatment consisted in daily haemodialysis and blood transfusions until stabilisation of the concentration of red blood cells and complementary sustainability management. The patient where stabilised, however, the renal function where no fully recovered.

Conclusion Although this pathology has a high mortality, the patient showed a favourable response to treatment, he was the only survivor of the three workers exposed directly to the gas arsine formation after adding zinc.

\section{CORRELATION BETWEEN CLINICAL ASSESSMENT OF PARKINSONISM, SELF-REPORTED SYMPTOMS AND MOTOR DYSFUNCTION IN A MANGANESE-EXPOSED COMMUNITY}

${ }^{1}$ Gill Nelson*, 1,2Brad Alan Racette. 'University of the Witwatersrand, Johannesburg, South Africa; ${ }^{2}$ Washington University School of Medicine, St. Louis, Missouri, USA

\subsection{6/oemed-2018-ICOHabstracts. 1450}

Introduction Exposure to high levels of manganese has been associated with progressive parkinsonism. Following complaints by residents in a South African town where a large manganese smelter operates, we designed a study to investigate neurological health effects (motor and cognitive) in adults. The objective of this analysis reported was to evaluate the correlation between parkinsonism and self-reported symptoms and health status, and fine-motor control. 
Methods Parkinsonism was assessed by a movement disorders specialist, using the Unified Parkinson Disease (PD) Rating Scale motor score (UPDRS3). The 39-item PD Questionnaire (PDQ-39) was used to assess participants' Parkinson diseasespecific quality of life. PD symptoms were self-reported, using a standard screening questionnaire. The grooved peg board timed test was used to measure fine motor speed and visuomotor coordination. We used locally weighted scatterplot smoothing (LOWESS) to graphically evaluate the associations of UPDRS3 score with age, grooved peg board times for both dominant and non-dominant hands, PDQ-39 score, and PD symptom questionnaire score. We also used LOWESS to evaluate the relationship between PDQ-39 score and symptom questionnaire score. We assessed correlations using Spearman coefficients.

Results The LOWESS plots and Spearman coefficients indicated positive associations $(\mathrm{p}<0.001)$, suggesting that individuals with higher UPDRS3 scores were older $(\rho=0.24)$, took longer to complete the grooved pegboard test (dominant $\rho=0.31$, non-dominant $\rho=0.28$ ), had higher PDQ-39 scores $(\rho=0.28)$, and had more PD symptoms $(\rho=0.35)$. Furthermore, PDQ-39 score was highly correlated $(\rho=0.70)$ with screening questionnaire score.

Discussion The strong correlations between parkinsonism and the administered tests showed that the tests used in this study are robust for identifying individuals with neurological health effects, are useful in large scale epidemiological studies, and may augment data obtained from a clinical specialist's examination.

\section{Unemployment and Job Insecurity}

\section{THE ASSOCIATION BETWEEN NOISE PERCEPTIONS WITH HEARING LOSS OCCURRENCE ON CARPENTERS OF INFORMAL SECTOR IN DUREN SAWIT DISTRICT, EAST JAKARTA}

SA Pitut*, Abdul Baktiansyah. The Faculty of Medicine and Health University of Muhammadiyah Jakarta

\subsection{6/oemed-2018-ICOHabstracts. 1451}

Background Industry's noise has long been an issue that cannot be resolved properly so it can be a serious threat to the workers's hearing function. In Indonesia, the incidence of noise-induced hearing loss is estimated between 20\%-30\% of the total working population in the formal sector who are productive, while the incidence of hearing loss due to noise in the informal sector is not yet known.

Objective This study aims to determine the relation of noise and other risk factors for hearing loss on carpenters of informal sector in Duren Sawit district, East Jakarta in 2013.

Method This study was an observational analytic with a cross sectional method. This research was carried out on 71 woodworkers in Duren Sawit districts, East Jakarta. Data were obtained from observations, additional physical examination, and an interview based on a questionnaire that has been made. Analysed using univariate and bivariate analysis.

Results A total of 51 workers $(71.8 \%)$ had subjective hearing loss. All workers have the perception that their workplace is quite noisy. In bivariate analysis, the use of Hearing Protection Devices has a significant effect on the occurrence of hearing loss, with a value of $\mathrm{p}=0.032, \mathrm{OR}=8.824$.
Conclusion Noise has a considerable impact on the occurrence of hearing loss. In addition, workers who did not use Hearing Protection Devices have 8 times greater risk for hearing loss compared with workers who use Hearing Protection Devices.

\section{EXPOSURE TO HAND-ARM VIBRATION AND RISK FACTORS FOR HAVS AMONGST OIL WORKERS IN THE UAE}

MA Ali". Emirates Integrated Telecommunications Company 'du', Dubai, United Arab Emirates (UAE)

\subsection{6/oemed-2018-ICOHabstracts. 1452}

Introduction There is a shortage of information on hand arm vibration syndrome (HAVS) data among exposed workers in developing countries, in particular hot regions. The oil industry in United Arab Emirates (UAE) is expanding and vibrating tools are used regularly in this industry. Data on the degree of exposure to hand arm vibration and associated symptoms among exposed workers in UAE are scarce.

Methods All workers $(n=115)$ exposed to hand-arm vibration in the company were included. Personal vibration levels were measured for vibration tools used for different job titles. Personal A (8) daily exposure values were calculated for all by combining typical daily exposure duration with measured tool vibration levels. A questionnaire was administered to all workers.

Results 5 participants were excluded due to Diabetes. Participants were divided in three exposure categories using the A (8) values. The prevalence of HAVS among the 110 participants was $13.6 \%$ (vibration white finger $0.9 \%$,neurosensory symptoms $3.6 \%$, and 9.1\% musculoskeletal symptoms). Cases of HAVS increased significantly with age, increasing exposure categories and total years vibration exposure. Multiple logistic regression analysis delineated that the only statistical significant predictor of HAVS was the current A (8) exposure level. Participants in highest exposure category were at a risk of HAVS 37 times greater than those in the lowest exposure category. (CI: 5-270.6).

Conclusion This is the first study of occupational exposure to vibration and risk factors for HAVS amongst oil workers in UAE. The study shows that the sensorineural and musculoskeletal components are more common then the vascular in warm area. Also HAVS symptoms were found to increase with increasing current A (8) exposure levels. It is essential that follow-up studies be carried out among larger numbers of handarm vibration exposed workers and appropriate health surveillance program developed to identify early sensorineural and musculoskeletal symptoms.

\section{VIBRATION EMISSION INFORMATION FOR USERS OF HAND-TOOLS IN THE EU AND NORTH AMERICA - AN INTERNATIONAL COMPARISON}

Eckardt Johanning. Columbia University; New York, New York, USA

\subsection{6/oemed-2018-ICOHabstracts. 1453}

Introduction The European Union (EU) Machinery Directive mandates that manufacturers inform the EU user of hand-held tools about the vibration values emitting acceleration exceeding $2.5 \mathrm{~m} / \mathrm{s}^{2} .^{(1)}$ Emission assessment and declaration guidelines exist (ISO 20643 and EN 60745). ${ }^{(2)}$ The goal of this study was to review and compare published manufacturer information for users 